\title{
Spatiotemporal Information Fusion for Fault Detection in Shipboard Auxiliary Systems
}

\author{
Soumalya Sarkar \\ Nurali Virani ${ }^{\dagger}$ \\ Murat Yasar ${ }^{\ddagger}$ \\ Asok Ray ${ }^{\dagger}$ \\ Soumik Sarkar ${ }^{\dagger}$ \\ svs5464@psu.edu \\ nnv105epsu.edu yasarmetechnosci.com \\ axr2epsu.edu \\ soumikpsu@gmail. com \\ $\dagger$ Department of Mechanical Engineering The Pennsylvania State University University Park, PA 16802, USA \\ $\ddagger$ Techno-Sciences, Inc. 11750 Beltsville Dr. Beltsville, MD 20705 USA
}

Keywords: Fault detection; Sensor fusion; Spatiotemporal patterns; Sensor network pruning; Symbolic Dynamics

\begin{abstract}
This paper addresses the issues of data analysis and sensor fusion that are critical for information management leading to (real-time) fault detection and classification in distributed physical processes (e.g., shipboard auxiliary systems). The proposed technique utilizes a semantic framework for multi-sensor data modeling, where the complexity is reduced by pruning the sensor network through an information-theoretic (e.g., mutual information-based) approach. The underlying algorithms are developed to achieve high reliability and computational efficiency while retaining the essential spatiotemporal characteristics of the physical system. The concept is validated on a simulation test bed of shipboard auxiliary systems.
\end{abstract}

\section{INTRODUCTION}

Sensor fusion has been one of the focused topics in data analysis and their applications. Individual sensory information is often used to reveal the underlying process dynamics and to identify potential changes therein. These systems are usually equipped with multiple sensors having (possibly) different modalities. These issues evince the need for the distributed and heterogeneous information to be fused to achieve accurate inferences about the states of critical systems in real time. To this end, this paper develops and validates a fault detection and classification method in distributed physical processes (e.g., shipboard auxiliary systems).

Several sensor fusion approaches have been proposed to address fault detection problems in the literature, including linear and nonlinear filters, adaptive model reference methodologies and neural network based estimation schemes. However, to the best of the authors' knowledge, these techniques have not been applied to shipboard systems due to several inherent difficulties that include: (possibly) non-stationary behavior of signals, underlying nonlinearities of the process dynamics, input-output and feedback interactions, scaling and alignment of multi-modal data, and multiplicative process noise.

Some of the above issues can be simplified to a certain extent or approximated by simpler solutions. Examples are linear and linearized modeling and Bayesian estimation techniques (e.g., extended Kalman filtering and particle filtering) [1][2]. Researchers have also used soft computing

This work has been supported in part by US Navy contract no. N00014M-12-0055. Any opinions, findings and conclusions or recommendations expressed in this publication are those of the authors and do not necessarily reflect the views of the sponsoring agencies. techniques; especially neural networks have been employed for sensor and actuator failure detection, identification and accommodation [3]. In the class of neural networks, multilayer perceptron [4] and radial basis function [5] configurations have been widely used for detection of anomalous patterns. Similarly, principal component analysis [6] and kernel regression [7] have been proposed for data-driven pattern classification. These approaches address nonlinear dynamics as well as scaling and data alignment issues. However, the effectiveness of data-driven techniques degrade rapidly in the case of multiplicative noise and extrapolating for non-stationary data. Robust filtering techniques have been developed and used to generate reliable estimations from sensors since most sensory data has some amount of noise in the measurement of the quantity [8], [9]. Recent literature has also reported Monte Carlo Markov chain techniques, such as particle filtering [10] and sigma point techniques [11] that yield numerical solutions to Bayesian state estimation problems and have been applied for nonlinear dynamical systems [12]. The estimation performance depends on the model accuracy which is the central problem with the filtering approach. Either the dynamics must be linear, or the data must be strictly periodic or stationary for the linear models to be good estimators. With increasing model fidelity and using nonlinear filters, the estimation error decreases considerably, however, these require numerical solutions that are computationally expensive and not suitable for real-time estimation. To address the problem of achieving reliable state estimation, many techniques have now appeared in literature, these include multiple model schemes [13], techniques based on analytical redundancy and residuals [14] and nonlinear observer theory [15].

For a general solution to be tractable for distributed systems (e.g., shipboard auxiliary systems), sensor fusion requires processing of non-stationary and nonlinear sensor data to detect parametric or nonparametric changes in the system. This paper presents the development of a sensor data fusion method for fault detection and classification in shipboard auxiliary systems. The proposed technique is built upon the algorithmic structure of symbolic dynamic filtering (SDF) [16], where a spatiotemporal pattern network is constructed from disparate sensors. The fully connected network is then pruned by applying an information-theoretic (e.g., 
mutual information-based) approach to reduce computational complexity. The developed algorithms are demonstrated on a notional electrical system coupled with a notional hydraulic system and a thermal load. A benchmark problem was created and the results under different performance metrics are presented.

The paper is organized in five sections including the present one. Section II explains the semantic framework for multi-sensor data modeling and the proposed technique to prune the heterogenous sensor network for information fusion. Section III describes the notional MATLAB/Simulink model to simulate Shipboard Auxiliary System. Section IV presents a fault injection scheme for the simulation and fault detection accuracy for different scenarios to validate the proposed method of information fusion. Finally, the paper is summarized and concluded in Section $\mathrm{V}$ with recommendations of future work.

\section{Semantic Framework for Multi-Sensor Data MODELING AND FUSION}

The semantic information fusion framework aims to capture temporal characteristics of individual sensor observations along with co-dependence among spatially distributed sensors. This paper presents a novel concept of Spatiotemporal pattern networks (STPNs) where temporal dynamics of each sensor as well as their relational dependencies are modeled as Probabilistic Finite State Automata (PFSA). Patterns emerging from individual sensors and relational dependencies are called atomic patterns (AP) and relational patterns (RP) respectively. In the STPN framework, sensors, APs and RPs can be thought of as nodes, self-loop links and link between two nodes respectively.

\section{A. Temporal Dynamics Modeling of Individual Sensor Data}

This section briefly describes the concepts of Symbolic Dynamic Filtering (SDF) for extracting atomic patterns from single-sensor data. The authors have explored the concepts of symbolic dynamics and time series data partitioning to develop this computationally efficient tool, for anomaly detection in complex dynamical systems [16].

Symbolic feature extraction from time series data is posed as a two-time-scale problem. The fast scale is related to the response time of the process dynamics. Over the span of data acquisition, dynamic behavior of the system is assumed to remain invariant, i.e., the process is quasi-stationary at the fast scale. On the other hand, the slow scale is related to the time span over which non-stationary evolution of the system dynamics may occur. It is expected that the features extracted from the fast-scale data will depict statistical changes between two different slow-scale epochs if the underlying system has undergone a change. The method of extracting features from stationary time series data is comprised of the following steps.

- Sensor time series data, generated from a physical system or its dynamical model, are collected at a slowscale epoch and let it be denoted as q. A compact (i.e., closed and bounded) region $\Omega \in \mathbb{R}^{n}$, where $n \in \mathbb{N}$, within which the stationary time series is circumscribed, is identified. Let the space of time series data sets be represented as $\chi \subseteq \mathbb{R}^{n \times T}$, where $T \in \mathbb{N}$ is sufficiently large for convergence of statistical properties within a specified threshold. While $n$ represents the dimensionality of the time-series, $T$ is the number of data points in the time series. Then, $\{\mathbf{s}\} \in \chi$ denotes a time series at the slow-scale epoch of data collection.

- Encoding of $\Omega$ is accomplished by introducing a partition $\mathbb{B} \triangleq\left\{B_{0}, \ldots, B_{(|\Sigma|-1)}\right\}$ consisting of $|\Sigma|$ mutually exclusive (i.e., $B_{j} \cap B_{k}=\emptyset \forall j \neq k$ ), and exhaustive (i.e., $\cup_{j=0}^{|\Sigma|-1} B_{j}=\Omega$ ) cells, where each cell is labeled by symbols $\sigma_{j} \in \Sigma$ and $\Sigma=\left\{\sigma_{0}, \ldots, \sigma_{|\Sigma|-1}\right\}$ is called the alphabet. This process of coarse graining can be executed by uniform, maximum entropy, or any other scheme of partitioning. Then, the time series data points that visit the cell $B_{j}$ are denoted as $\sigma_{j} \forall j=$ $0,1, \ldots,|\Sigma|-1$. This step enables transformation of the time series data $\{\mathbf{s}\}$ to a symbol sequence $\{\mathbf{s}\}$, consisting of the symbols $\sigma_{j}$ in the alphabet $\Sigma$.

- A probabilistic finite state automata $(P F S A)$, is then constructed. The PFSA considered in this framework is known as D-Markov machine [16]. Formally, a state in a D-Markov machine is a symbol sequence of length $D$. States represent all possible words of length $\mathrm{D}$, using the symbol alphabet. With this setup, $\tilde{\Pi}$ denotes the symbol generation matrix elements of which are the probabilities of obtaining a symbol conditioned on current state of the system. Similarly, $\Pi$ denotes the state transition matrix that captures the state transition probabilities. Note, $\Pi$ and $\tilde{\Pi}$ are same for $D=1$.

Let the state of a sensor $A$ at the $k^{\text {th }}$ instant be denoted as $q_{k}^{A}$. With this notation, the $i j^{t h}$ matrix element $\pi_{i j}^{A}$ of the state transition matrix is defined as the probability that $(n+1)^{t h}$ state is $i$ given that the $n^{t h}$ state was $j$, i.e.,

$$
\pi_{i j}^{A} \triangleq P\left(q_{n+1}^{A}=i \mid q_{n}^{A}=j\right)
$$

\section{B. Spatiotemporal Pattern Analysis from Multi-sensor Data}

To construct STPN, we need relational patterns which are essentially extracted from the relational probabilistic finite state automata (PFSA). These are discovered using $\mathrm{xD}-\mathrm{Markov}$ machine construction to determine crossdependence; the algorithm is described in Section II-B.1.

1) Construction of relational PFSA: xD-Markov machine: This section describes the construction of $\mathrm{xD}$-Markov machines from two symbol sequences $\left\{\mathbf{s}_{1}\right\}$ and $\left\{\mathbf{s}_{2}\right\}$ obtained from two different sensors (possibly of different modalities) to capture the symbol level cross-dependence. A formal definition is as follows:

Definition II.1 (xD-Markov) Let $\mathcal{M}_{1}$ and $\mathcal{M}_{2}$ be the PFSAs corresponding to symbol streams $\left\{\boldsymbol{s}_{1}\right\}$ and $\left\{\boldsymbol{s}_{2}\right\}$ respectively. Then a $x D$-Markov machine is defined as a 5-tuple $\mathcal{M}_{1 \rightarrow 2} \triangleq\left(\mathcal{Q}_{1}, \Sigma_{1}, \Sigma_{2}, \delta_{1}, \tilde{\Pi}_{12}\right)$ such that:

- $\Sigma_{1}=\left\{\sigma_{0}, \ldots, \sigma_{\left|\Sigma_{1}\right|-1}\right\}$ is the alphabet set of symbol sequence $\left\{\boldsymbol{s}_{1}\right\}$ 
- $\mathcal{Q}_{1}=\left\{q_{1}, q_{2}, \ldots, q_{|\Sigma|_{1}^{D_{1}}}\right\}$ is the state set corresponding to symbol sequence $\left\{\boldsymbol{s}_{1}\right\}$, where $D_{1}$ is the depth for $\left\{\boldsymbol{s}_{1}\right\}$

- $\Sigma_{2}=\left\{\sigma_{0}, \ldots, \sigma_{\left|\Sigma_{2}\right|-1}\right\}$ is the alphabet set of symbol sequence $\left\{\boldsymbol{s}_{2}\right\}$

- $\delta_{1}: \mathcal{Q}_{1} \times \Sigma_{1} \rightarrow \mathcal{Q}_{1}$ is the state transition mapping that maps the transition in symbol sequence $\left\{\boldsymbol{s}_{1}\right\}$ from one state to another upon arrival of a symbol in $\left\{\boldsymbol{s}_{1}\right\}$

- $\tilde{\Pi}_{12}$ is the symbol generation matrix of size $\left|\mathcal{Q}_{1}\right| \times\left|\Sigma_{2}\right|$; the $i j^{\text {th }}$ element of $\tilde{\Pi}_{12}$ denotes the probability of finding $j^{\text {th }}$ symbol in $\left\{\boldsymbol{s}_{2}\right\}$ while making a transition from $i^{\text {th }}$ state in the symbol sequence $\left\{\boldsymbol{s}_{1}\right\}$

In practice, $\tilde{\Pi}_{12}$ is reshaped into a vector of length $\left|\mathcal{Q}_{1}\right| \times\left|\Sigma_{2}\right|$ and is treated as the extracted feature vector that is a low-dimensional representation of the relational dependence between $\left\{\mathbf{s}_{1}\right\}$ and $\left\{\mathbf{s}_{2}\right\}$. This feature vector is called a Relational Pattern (RP). Note, when both symbol sequences are same, the relational patterns are essentially the atomic pattern corresponding to the symbol sequence; i.e., xD-Markov machine reduces to a simple D-Markov machine. Also, a Relational Pattern between two symbol sequences is not necessarily symmetric; therefore, RPs need to be identified for both directions. In this analysis we need state transition matrices which captures the state level cross-dependence between sensors. As illustrated in Fig. 1, elements of the state transition matrices $\Pi^{A B}$ and $\Pi^{B A}$ corresponding to the cross machines can be expressed as,

$$
\begin{aligned}
& \pi_{i l}^{A B} \triangleq P\left(q_{n+1}^{B}=l \mid q_{n}^{A}=i\right) \\
& \pi_{k j}^{B A} \triangleq P\left(q_{n+1}^{A}=j \mid q_{n}^{B}=k\right)
\end{aligned}
$$

where, $i, j \in Q^{A}$ and $k, l \in Q^{B}$. For depth $D=1$ state and symbol of a D-Markov machine carry same meaning. This study limited the depth to 1 , which allowed us to use relational pattern $\tilde{\Pi}_{12}$ and state transition matrix $\Pi^{12}$ interchangeably as feature. One of the important future scopes of this methodology includes the construction of state transition matrix from xD-Markov machine for depth $D>1$.

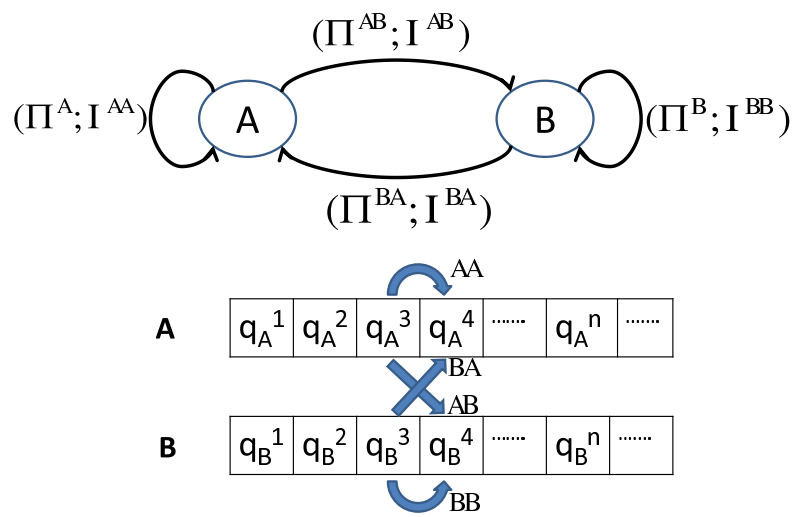

Fig. 1: Illustration of Spatiotemporal Pattern Network (STPN)
2) Pruning of STPN: From a system perspective, all the atomic and relational patterns need to be considered in order to model the nominal behaviors or detect anomalies. However, it is obvious that there is scalability issue associated with systems with significant number of sensors as the number of relational patterns increases quadratically with number of sensors (number of RPs will be $S(S-1$ ) where, $S$ is the total number of sensors; thus, total number of patterns will be $S^{2}$ ). This pattern space explosion may prohibit use of a complete STPN approach for monitoring of large systems under computational and memory constraints. Moreover, it is understood that for many real systems, a large fraction of relational patterns may have a very low information content due to the lack of their physical (electro-mechanical or via control loop) dependencies. Therefore, a pruning process needs to be established to identify a sufficient STPN for a system. This paper adopts an Information-theoretic measure involving Mutual Information to identify the importance of an atomic or a relational pattern. Mutual information based criteria have been very popular and useful in general graph pruning strategies [17], [18] including structure learning of Bayesian Networks [19]. In the present context, mutual information essentially provides the information content of an atomic or a relational pattern. The network pruning strategy is described below:

Mutual information for the Spatiotemporal pattern of sensor $X$ and $Y$ is expressed as,

$$
I^{X Y}=I\left(q_{n+1}^{Y} ; q_{n}^{X}\right)=H\left(q_{n+1}^{Y}\right)-H\left(q_{n+1}^{Y} \mid q_{n}^{X}\right)
$$

where,

$$
\begin{gathered}
H\left(q_{n+1}^{Y}\right)=-\sum_{i=1}^{Q_{Y}} P\left(q_{n+1}^{Y}=i\right) \log _{2} P\left(q_{n+1}^{Y}=i\right) \\
H\left(q_{n+1}^{Y} \mid q_{n}^{X}\right)=-\sum_{i=1}^{Q_{X}} P\left(q_{n}^{X}=i\right) H\left(q_{n+1}^{Y} \mid q_{n}^{X}=i\right) \\
H\left(q_{n+1}^{Y} \mid q_{n}^{X}=i\right)=-\sum_{i=1}^{Q_{Y}} P\left(q_{n+1}^{Y}=l \mid q_{n}^{X}=i\right) . \\
\log _{2} P\left(q_{n+1}^{Y}=l \mid q_{n}^{X}=i\right)
\end{gathered}
$$

When $X=Y=A, I^{X Y}$ is the Mutual information for atomic pattern for the sensor A. The quantity $I^{A A}$ essentially captures the temporal self-prediction capability (self-loop) of sensor $A$. As an extreme example, the atomic pattern for a random sensor data is not very informative and its self mutual information will also be zero (under ideal estimation).

When $X=A$ and $Y=B, I^{X Y}$ is the Mutual information for the relational pattern $R^{A B}$. The quantity $I^{A B}$ essentially captures sensor $A$ 's capability of predicting sensor $B$. Similar to atomic patterns, an extreme example would be the scenario where sensor $A$ and $B$ are not co-dependent (i.e., sensor $A$ completely fails to predict temporal evolution of sensor $B$ ). In this case, $R^{A B}$ is not very informative and $I^{A B}$ will also be zero (under ideal estimation). 
The next step is to select a fraction of patterns from the entire pool based on some threshold on a measure of information gain due to atomic and relational patterns. Let $\mathcal{S}$ is set of all sensors. Now, the goal is to accept some patterns from the set of $\mathcal{S} \times \mathcal{S}$. Let set of accepted patterns be denoted as $\mathcal{P}^{\text {accept }} \subset \mathcal{S} \times \mathcal{S}$ and the corresponding information gain be denoted as $I_{G}^{a c c e p t}$. The accept pattern set is chosen such that, for a $\eta \in[0,1]$

$$
\frac{I_{G}^{a c c e p t}}{\sum_{(A, B) \in \mathcal{S} \times \mathcal{S}} I^{A B}} \geq \eta
$$

$\eta$ is chosen as 0.9 for the validation experiments. Note, in this pruning strategy all patterns related to a certain sensor may get rejected. Therefore, the user may choose to put additional constraint of keeping at least one (atomic or relational) pattern for each sensor in the accepted set of patterns.

Remark II.1 In order to use the STPN for fault detection, a network of PFSA can be identified following the above process under the nominal condition. Faulty conditions can then be detected by identifying the changes in parameters related to the accepted patterns. However, under some severe faults, the causal dependency characteristics (i.e., the structure itself) among nodes may not remain invariant. In such cases, new structures of the STPN can signify the severely faulty conditions.

\section{Description OF Simulation TEST BeD}

For testing the proposed algorithm of sensor fusion in a coupled, distributed environment, a simulation of a notional hydraulic system coupled with a notional electrical system and a thermal load is developed. The simulation model is implemented in MATLAB/Simulink as seen in Fig. 2.

This distributed notional system is driven by the external speed command, $\omega_{r e f}$, that is used as a set point for the speed, $\omega_{e}$, of the permanent magnet synchronous motor (PMSM). A mechanical shaft coupling connects the fixed displacement pump (FDP) to the PMSM. The torque load of the PMSM, $T_{m}$, is obtained from the shaft model of the hydraulic system. In turn, the speed of the PMSM, $\omega_{e}$, is an input to determine the speed of the shaft, $\omega_{s}$, that drives the FDP and the cooling fan in the thermal system. In turn, the FDP drives the hydraulic motor (HM) with a dynamic load, which consists of the thermal load, $T_{t}$, and a timevarying mechanical torque load. The PMSM of the electrical

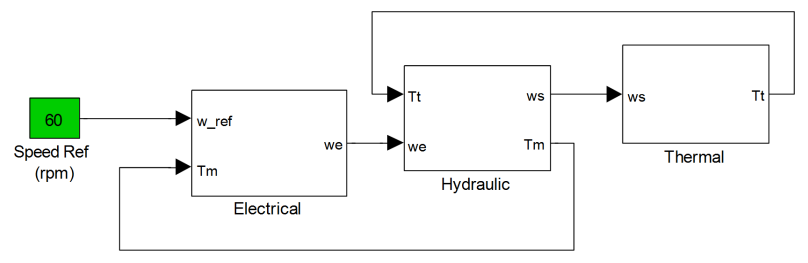

Fig. 2: Notional coupled electrical, hydraulic and thermal systems.

system is powered by a voltage source that drives the IGBT using the Hall Effect sensors of the PMSM. The PI controller regulates the motors electrical frequency under dynamic loading conditions that arise due to hydraulic fluctuations and thermal effects. A mechanical coupling connects the PMSM to the FDP of the hydraulic system. This is modeled by a rotational spring-damper system applied to an inertial load. The mechanical system outputs the shaft velocity that drives the FDP and the cooling fan of the thermal system. The pump, in turn, powers the HM through the pipeline. The HM is subjected to a time-varying load with a profile defined by the user as well as the thermal load that varies with the fan efficiency of the cooling mechanism. The systems are further coupled with a feedback loop since the torque requirement of the HM is input to the PMSM of the electrical system. The model has multiple parameters that can simulate various fault conditions. There are multiple sensors in each system with different modalities such as Hall Effect sensors, torque, speed, current, temperature and hydraulic pressure sensors which will be explained further in section IV.

\section{VALIDATION EXPERIMENTS AND RESUlTS}

This section validates the pruning technique of STPN based on mutual information and fuse useful patterns from heterogenous sensors to identify the faults in the distributed system described in section III.

\section{A. Fault Injection, Sensors, Data Partitioning}

This system has Electrical, Hydraulic and Thermal subsystems. Each sub-system has a set of sensors as given in Table I to observe the system behavior. Also, each subsystem has parameters which can be changed to induce faults as given in Table II. The applicability of the proposed technique has been justified in this paper under the assumptions mentioned below.

- At any point of time, the system can have only one of the faults mentioned in Table II because the occurrence of two faults simultaneously is very unlikely in real scenarios.

- Mechanical efficiency of Hydraulic Motors and Pumps is assumed to stay constant as the degradation of machines due to wear and tear is at a much slower rate with respect to the drop in volumetric efficiency.

- The model is equipped with commercially available sensors. Exploration of other feasible sensors to help improve fault detection is not the focus of this study.

Fig. 3 depicts a typical electromagnetic torque output for nominal and PMSM fault cases. As it can be seen that the data itself is very noisy and due to the controller actions there is not much observable difference in these two cases. To enhance the fault identification accuracy in these types of critical fault scenarios, information integration from disparate sensors is performed. For all the fault scenarios 100 samples from every sensor are divided into two equal halves for training and testing. For data partitioning, maximum entropy partitioning is used with alphabet size, $|\Sigma|=6$ for seven sensors (although $|\Sigma|$ does not need to be same for different sensors). The depth for constructing PFSA states is taken to be, $D=1$ for both atomic pattern and relational pattern construction. A reduced set of these patterns are aggregated 
TABLE I: Sensors of the system

\begin{tabular}{c|ccc}
\hline System & Sensor & Physical Quantity & Symbol \\
\hline Electrical & Torque sensor & Torque output of PMSM & $T_{e}$ \\
& Rotational Motion Sensor & Rotor speed of PMSM & $w_{e}$ \\
Hydraulic & Rotational Motion Sensor & Angular Velocity of Hydraulic Pump & $w_{h p}$ \\
& Pressure Sensor & Pressure across Hydraulic Motor (HM) & $P_{h m}$ \\
& Torque sensor & Torque output of HM & $T_{h m}$ \\
& Rotational Motion Sensor & Angular Velocity of output shaft of Hydraulic Motor & $w_{h m}$ \\
Thermal & Temperature Sensor & Temperature & $T_{f}$ \\
\hline
\end{tabular}

TABLE II: Fault parameters of the system

\begin{tabular}{c|ccc}
\hline System & Fault parameter & Symbol & Range \\
\hline Electrical & Flux linkage of PMSM & $W_{b}$ & Nominal:0.05 \pm 0.005, Fault:0.03 \pm 0.005 \\
Hydraulic & Volumetric Efficiency of HM & $\nu_{v m}$ & Nominal:0.9 \pm 0.02, Fault:0.8 \pm 0.02 \\
& Total Efficiency of HM & $\nu_{t m}$ & Nominal:0.8 \pm 0.02, Fault:0.65 \pm 0.02 \\
Thermal & Thermal efficiency & $\nu_{t h}$ & Nominal:0.9 \pm 0.02, Fault:0.8 \pm 0.02 \\
\hline
\end{tabular}

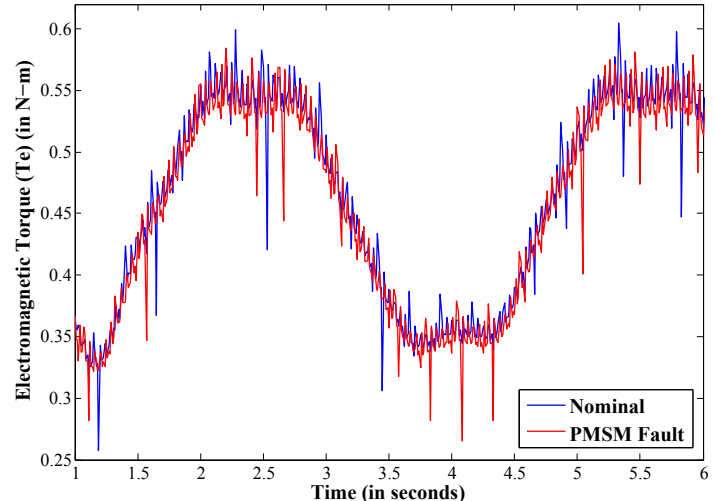

Fig. 3: Electromagnetic torque under nominal and PMSM fault conditions

to form the composite pattern, which serves as the feature classified by a k-NN classifier (with $k=5$ ) using the Euclidean distance metric for fault detection.

\section{B. Fusion with Complete STPN}

One sensor from each of the subsystems, i.e., $T_{f}$ from thermal subsystem, $T_{e}$ from electrical subsystem and $T_{h m}$ from hydraulic subsystem are selected to perform fusion to identify component faults in the system. The Composite Pattern (CP) consists of all possible Atomic Patterns (AP) and Relational Patterns (RP) of $T_{f}, T_{e}$ and $T_{h m}$. Composite TABLE III: Fault identification accuracy by exhaustive fusion

\begin{tabular}{|c|cccc|}
\hline class & $T_{f}$ & $T_{e}$ & $T_{h m}$ & $\mathrm{CP}$ \\
\hline Nominal & $32 \%$ & $42 \%$ & $32 \%$ & $68 \%$ \\
PMSM fault & $30 \%$ & $100 \%$ & $40 \%$ & $84 \%$ \\
HM fault & $40 \%$ & $100 \%$ & $100 \%$ & $100 \%$ \\
Thermal fault & $100 \%$ & $58 \%$ & $44 \%$ & $100 \%$ \\
\hline
\end{tabular}

pattern (CP) performs better in nominal condition detection than individual sensors as shown in Table III, but the false alarm rate is still high. Composite patterns are formed by concatenating atomic and relational patterns. Therefore, while patterns with high information content (based on the formulation above) help distinguishing between classes, patterns with low information content dilutes the ability of separating classes. Therefore, removing non-informative patterns may lead to reduction of both false alarm, missed detection rates and computational complexity.

\section{Pruning of STPN}

Pruning of the larger sensor network of the given system is attempted here to reduce the complexity of fusion and improve the detection accuracy by capturing the essential spatiotemporal dynamics of the system. Left half of the Fig. 4 shows a fully connected graph of seven sensors of the system where each node is a sensor; bi-directional arcs among them depict the RPs in both directions and self-loops are the APs corresponding to sensors. The right half of the

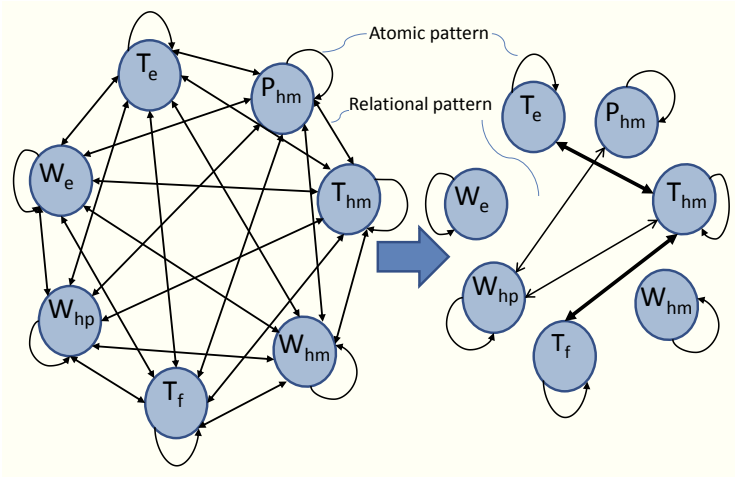

Fig. 4: Pruning of STPN

Fig. 4 demonstrates the pruned STPN where the thickness of arcs represents the intensity of mutual information of the RPs among sensors. Both directions of arrows are preserved as the mutual information of the two oppositely directed RPs for a pair of sensors are comparable. In this example all the self loops are kept intact and arcs with negligible mutual information are omitted from the graph. it is observed in this study, that the structure of the reduced STPN remains stable across all the fault classes. The reduction in complexity of network graph is more significant in larger STPN.Two scenarios are as follows to justify the credibility of the pruned STPN in the light of fault detection accuracy.

1) Reduction of false alarms: Same set of sensors, i.e., $T_{f}, T_{e}$ and $T_{h m}$ are selected as the STPN and it is subjected to the proposed pruning technique, which results in a composite pattern of AP of $T_{e}\left(\Pi^{T_{e}}\right)$ and two RPs $\left(\Pi^{T_{h m} T_{e}}\right.$, $\Pi^{T_{h m} T_{f}}$ shown by two thick arcs in Fig. 4) reducing the false alarm rate significantly (Table. IV). APs of $T_{f}$ and $T_{h m}$ are dropped from the CP as these patterns don't add to better detection. Also PMSM fault detection accuracy 
TABLE IV: Comparison of false alarm rate generated by exhaustive sensor fusion and pruned STPN

\begin{tabular}{c|c}
\hline Fusion type & False alarm rate \\
\hline complete STPN & $32 \%$ \\
Pruned STPN & $8 \%$ \\
\hline
\end{tabular}

doesn't degrade from $100 \%$ unlike fusion with complete STPN. Hence this pruning technique reduces a CP containing 9 patterns (3 APs, 6 RPs)to a CP of three APs and two RPs along with providing more class separability.

2) Adaptability to malfunctioning sensors: In a distributed system, such as shipboard auxiliary system, malfunctioning of primary sensors in a subsystem is a plausible event. One of the current challenges in fault detection area is to identify any fault in the subsystem with malfunctioning sensors from the sensor responses of the subsystems electromechanically connected to that. To simulate that situation three prime heterogenous sensors from the hydraulic subsystem, i.e., $w_{h p}, P_{h m}$ and $T_{h m}$ are selected whereas fault is injected to the thermal subsystem by degrading the thermal efficiency according to Table. II. $T_{f}$ sensor of thermal subsystem is not incorporated in the detection process of thermal fault as it considered to be the malfunctioning sensor.

TABLE V: Thermal fault identification by sensors of hydraulic subsystem

\begin{tabular}{c|cccc}
\hline & $w_{h p}$ & $P_{h m}$ & $T_{h m}$ & $\mathrm{CP}$ \\
\hline Detection accuracy & $58 \%$ & $18 \%$ & $18 \%$ & $70 \%$ \\
\hline
\end{tabular}

As the individual sensors of hydraulic subsystem perform poorly in detecting thermal fault (Table. V) information from sensors of this subsystem are fused by the proposed pruning technique. The pruned STPN yields a composite pattern consisting of AP of $w_{h p}\left(\Pi^{w_{h p}}\right)$ and two RPs $\left(\Pi^{P_{h m} w_{h p}}\right.$, $\Pi^{T_{h m} w_{h p}}$ ) depicted by two thin arcs in Fig. 4; it results in a decent detection accuracy of $70 \%$ (Table V).

\section{COnClusions And Future Work}

This paper deals with the issue of feature level fusion of multiple sensor data for data-driven fault detection techniques. The underlying algorithms are built upon the concepts of symbolic dynamic filtering (SDF) to construct a spatiotemporal pattern network from disparate sensors. The fully-connected network is pruned by applying an information theoretic approach to reduce computational complexity. In the proposed method, the abstract semantic fusion framework captures the temporal characteristics of individual sensor observations (Atomic Patterns) along with co-dependence among spatially distributed sensors (Relational Patterns) and constructs a fully connected graph of the sensor network. The pruning strategy preserves the patterns containing higher mutual Information to construct the composite pattern that serves as the primary feature for fault detection in real time. The validation of the proposed method on a test bed representing shipboard auxiliary systems shows that fusion with network pruning identifies component faults with better accuracy than fusion with fully-connected sensor network.
Confidence assessment of the pruned network in representing the distributed fully-connected sensor network is the topic of the present work. In addition, the following research areas are currently being pursued.

- Optimization of the threshold of ratio of mutual information $\eta$ (Section II-B.2) subjected to better fault detection and lesser complexity.

- Rigorous testing of robustness of the pruning strategies over different fault classes.

- Validation of the fusion algorithm on larger sensor network of real distributed system.

- Comparison between developed method and other stateof-the-art network pruning and fusion algorithm (both model-driven and data-driven) for fault detection.

\section{REFERENCES}

[1] D. L. Simon and S. Garg, "Optimal tuner selection for kalman filterbased aircraft engine performance estimation," Journal of Engineering for Gas Turbines and Power, vol. 132, p. 031601, 2010.

[2] A. Doucet, N. de Freitas, and N. Gordon, Sequential Monte Carlo Methods in Practice. New York, NY, USA: Springer, 1 ed., 2001.

[3] M. R. Napolitano, Y. An, and B. A. Seanor, "A fault tolerant flight control system for sensor and actuator failures using neural networks," Aircraft Design, vol. 3, pp. 103-128, 2000.

[4] J. Liu and J. M. A. Scherpen, "Fault detection method for nonlinear systems based on probabilistic neural network filtering," International Journal of Systems Science, vol. 33, pp. 1039-1050, 2002.

[5] S. Haykin, Neural Networks : A Comprehensive Foundation. Upper Saddle River, NJ: Prentice Hall, 1999.

[6] K. Fukunaga, Statistical Pattern Recognition. Boston, MA, USA: Academic Press, 2 ed., 1990.

[7] J. Shawe-Taylor, Kernel Methods for Pattern Analysis. Cambridge, U.K.: Cambridge University Press, 2004.

[8] M. S. Grewal and A. P. Andrews, Kalman Filtering: Theory and Practice Using MATLAB. John Wiley \& Sons, 2 ed., 2001.

[9] A. Gelb, ed., Applied Optimal Estimation. The MIT Press, 1974.

[10] C. Andrieu, A. Doucet, S. Singh, and V. B. Tadic, "Particle methods for change detection, system identification, and control," Proceedings of IEEE, vol. 92, pp. 423-438, 2004.

[11] S. Julier, J. Uhlmann, and H. F. Durrant-Whyte, "A new method for the nonlinear transformation of means andcovariances in filters and estimators," IEEE Transactions on Automatic Control, vol. 45, pp. 477-482, 2000.

[12] P. Li and V. Kadirkamanathan, "Particle filtering based likelihood ratio approach to fault diagnosis in nonlinear stochastic systems," IEEE Transactions on Systems, Man and Cybernetics, vol. 31, pp. 337-343, 2001.

[13] M. Gopinathan, J. D. Boskovic, R. K. Mehra, and C. Rago, "A multiple model predictive scheme for fault-tolerant flight control design," in Conference on Decision and Control, pp. 1376-1381, 2001.

[14] J. J. Gertler, "Survey of model-based failure detection and isolation in complex plants," IEEE Control Systems Magazine, vol. 8, pp. 3-11, 1988.

[15] E. A. Garcia and P. M. Frank, "Deterministic nonlinear observerbased approaches to fault diagnosis: A survey," Control Engineering Practice, vol. 5, pp. 663-670, 1997.

[16] A. Ray, "Symbolic dynamic analysis of complex systems for anomaly detection," Signal Processing, vol. 84, pp. 1115-1130, 2004.

[17] H. Kretzschmar, C. Stachniss, and G. Grisetti, "Efficient informationtheoretic graph pruning for graph-based slam with laser range finders," in International Conference on Intelligent Robots and Systems (IROS), pp. $865-871,2011$.

[18] A. J. Butte and I. S. Kohane, "Mutual information relevance networks:functional genomic clustering using pairwise entropy measurements," in Pacific Symposium on Biocomputing, pp. 418-429, 2000.

[19] L. M. de Campos, "A scoring function for learning bayesian networks based on mutual information and conditional independence tests," Journal of Machine Learning Research, vol. 7, pp. 2149-2187, 2006. 